\title{
Asymmetric neutrino emission due to neutrino-nucleon scatterings in supernova magnetic fields
}

\author{
Shin'ichiro Andd* \\ Department of Physics, School of Science, \\ The University of Tokyo, 7-3-1 Hongo, \\ Bunkyo-ku, Tokyo 113-0033, Japan
}

(Dated: June 19, 2018)

\begin{abstract}
We derive the cross section of neutrino-nucleon scatterings in supernova magnetic fields, including weak-magnetism and recoil corrections. Since the weak interaction violates the parity, the scattering cross section asymmetrically depends on the directions of the neutrino momenta to the magnetic field; the origin of pulsar kicks may be explained by the mechanism. An asymmetric neutrino emission (a drift flux) due to neutrino-nucleon scatterings is absent at the leading level of $\mathcal{O}\left(\mu_{B} B / T\right)$, where $\mu_{B}$ is the nucleon magneton, $B$ is the magnetic field strength, and $T$ is the matter temperature at a neutrinosphere. This is because at this level the drift flux of the neutrinos are exactly canceled by that of the antineutrinos. Hence, the relevant asymmetry in the neutrino emission is suppressed by much smaller coefficient of $\mathcal{O}\left(\mu_{B} B / m\right)$, where $m$ is the nucleon mass; detailed form of the relevant drift flux is also derived from the scattering cross section, using a simple diffusion approximation. It appears that the asymmetric neutrino emission is too small to induce the observed pulsar kicks. However, we note the fact that the drift flux is proportional to the deviation of the neutrino distribution function from the value of thermal equilibrium at neutrinosphere. Since the deviation can be large for non-electron neutrinos, it is expected that there occurs cancellation between the deviation and the small suppression factor of $\mathcal{O}\left(\mu_{B} B / m\right)$. Using a simple parameterization, we show that the drift flux due to neutrino-nucleon scatterings may be comparable to the leading term due to beta processes with nucleons, which has been estimated to give a relevant kick velocity when the magnetic field is sufficiently strong as $10^{15}-10^{16} \mathrm{G}$.
\end{abstract}

PACS numbers: 97.60.Bw, 13.15.+g, 11.30.Er

*Email address: ando@utap.phys.s.u-tokyo.ac.jp 


\section{INTRODUCTION}

A core-collapse supernova explosion is one of the most spectacular events in astrophysics; $99 \%$ of its gravitational binding energy is released as neutrinos, while only $1 \%$ as the kinetic energy of a shock wave. Therefore, neutrinos play an essential role in supernova explosions, and their detection by ground-based large water Čherenkov detectors, such as Super-Kamiokande and Sudbury Neutrino Observatory, would provide valuable information on the nature of neutrinos as well as supernova physics (see Ref. [1], and references therein).

Because of their dominance in supernova energetics, the neutrinos may also give the solution to a long-standing astrophysical mystery, or "pulsar kicks." Recent analyses of pulsar proper motion give a mean birth velocity 200-500 $\mathrm{km} \mathrm{s}^{-1}$ [2, 3, 4, 5], 6], with possibly a significant population having velocity $\gtrsim 1000 \mathrm{~km} \mathrm{~s}^{-1}$. These values are much greater than the velocities of their progenitors $\left(\sim 30 \mathrm{~km} \mathrm{~s}^{-1}\right)$. A natural explanation for such high velocities is that supernova explosions are asymmetric, and provide kicks to nascent neutron stars. In this paper, we are concerned with models in which the pulsar kicks arise from magnetic field induced asymmetry in neutrino emissions from proto-neutron stars. We do not deal with another type of mechanism which relies on hydrodynamical instabilities in the collapsed stellar core [7, 8, 9, 10, 11], leading to asymmetric matter ejection and/or asymmetric neutrino emission. Concerning the latter mechanism, several numerical results gave rather negative results 9 , 12].

There are many past studies which have noted that parity violation in the weak interaction can lead to asymmetric neutrino emission in the strongly magnetized neutron star matter (see, e.g., Refs. [13, 14, 15]). Arras and Lai [14] have shown that an asymmetric neutrino emission (a drift flux) that may give the pulsar kicks is mainly induced by charged-current interactions with nucleons, $\nu_{e} n \rightarrow e^{-} p$ and $\bar{\nu}_{e} p \rightarrow e^{+} n$, in magnetic fields. On the other hand, the asymmetric emissions due to neutral-current interactions, $\nu N \rightarrow \nu N$, are found to be irrelevant because their contributions cancel between neutrinos and antineutrinos. Further their finding is that the drift flux exists only when the distribution deviates significantly from the value of thermal equilibrium. This result has invalidated the previous optimistic estimation that sufficient asymmetry can be obtained by multiple scatterings of neutrinos by nucleons, slightly polarized by the magnetic field of moderate strength $\left(\sim 10^{12} \mathrm{G}\right)$ [15]. Their naive estimation has shown that the asymmetry parameter for the $\nu_{e}, \bar{\nu}_{e}$ flux would be 
sufficient for the observed pulsar kick velocity, if the magnetic field is very strong, $B \sim 10^{15}$ $10^{16} \mathrm{G}$.

In the present study, we focus on the effect of weak magnetism and recoil corrections on neutrino-nucleon scatterings (particularly $\nu_{\mu, \tau^{-}} N$ and $\bar{\nu}_{\mu, \tau^{-}} N$ ) in supernova magnetic fields, which has not been considered in the previous publications such as Ref. [14]. We expect that including these two corrections changes the previous conclusions, which are listed above, as follows. The weak magnetism correction reduces antineutrino-nucleon cross sections compared to those of neutrino-nucleon scatterings [16]. For the reason, the cancellation of asymmetry between $\nu$ and $\bar{\nu}$, which were shown in Ref. [14], does not occur at $\mathcal{O}(k / m)$ level, where $k$ is the neutrino energy and $m$ is the nucleon mass. Although the drift flux due to $\nu$-N scatterings is suppressed by a small factor of $\mathcal{O}(k / m)$, this term may be as large as the term due to charged-current interactions between electron (anti)neutrinos and nucleons. This is because the drift flux is proportional to the deviations of distribution functions from the value of thermal equilibrium at a neutrinosphere [14]. ${ }^{1}$ For $\mu$ and $\tau$ neutrinos, with which we are concerned in this study, the transport opacity is primarily resulted from $\nu$ - $N$ scattering, whereas energy exchange is due to elastic $\nu$ - $e^{-}$scattering whose cross section is substantially smaller. As a consequence, the decoupling layer locates at much deeper region than the neutrinosphere and there should be large optical depth for the asymmetric flux to develop. On the other hand, for $\nu_{e}$ and $\bar{\nu}_{e}$, the difference from equilibrium at the neutrinosphere is considered to be very small owing to dominant $\nu_{e} n \rightarrow e^{-} p$ and $\bar{\nu}_{e} p \rightarrow e^{+} n$ reactions, which act as very efficient energy-exchanging and thermalizing reactions. Thus, although the drift flux due to $\nu_{\mu, \tau}$ and $\bar{\nu}_{\mu, \tau}$ is suppressed by a small coefficient of $\mathcal{O}(k / m)$ compared with that due to $\nu_{e}$ and $\bar{\nu}_{e}$, we believe that the large deviations from equilibrium for non-electron neutrinos can counter the suppression factor.

Motivated by the above reasoning, we first derive the cross section for $\nu$ - $N$ scatterings in magnetic field by including the weak magnetism and recoil corrections of scattered nucleons. In general, it is quite difficult to evaluate. However, in the limit that the nucleons are nondegenerate, we can present a simple form of the differential cross section, and give a diffusion equation which enables us to discuss qualitatively whether the effect we are

\footnotetext{
${ }^{1}$ In this paper, we use a term "neutrinosphere" as that above which neutrinos freely stream out, while by terms such as "decoupling surface," we intend the surface within which neutrinos are kept in thermal equilibrium.
} 
tackling is important or not. We show, as a result of the diffusion equation and a simple parameterization, that the drift flux on which we concentrate can contribute at a considerable level for reasonable parameter choice.

Throughout this paper, we do not consider the effect of neutrino oscillation, although recent atmospheric [17], solar [18], and reactor experiments [19] have shown that the neutrinos have the nonzero masses and mixings. This treatment is justified because the flavor mixing between electron and the other flavor neutrinos is strongly suppressed by matter effect. Roughly speaking, the efficient flavor conversion takes place at a so-called resonance region, where a condition $n_{e} \simeq \Delta m^{2} / G_{F} k$ is satisfied, however, at much deeper region $n_{e} \gg \Delta m^{2} / G_{F} k$ matter induced neutrino oscillation is highly suppressed (see, e.g., Ref. [20]). With the recently inferred parameters $\Delta m^{2}$ by the oscillation experiments, the resonance region locates at rather outer envelope such as $\mathrm{O}+\mathrm{C}$ or He layers; at a region around the neutrinosphere on which we are focusing in this study, matter potential strongly prevents electron (anti)neutrinos from mixing with the other flavor (anti)neutrinos. In other words, at the sufficiently deep region in stars, electron (anti)neutrinos propagate as the mass-eigenstates. For $\mu$ and $\tau$ flavor (anti)neutrinos, they are found maximally mixing with each other, however, it does not affect the supernova dynamics such as pulsar kicks, because they can not be distinguished in supernovae. Thus, there is no need to worry about the effects of neutrino oscillation on supernova dynamics. Several years ago, Kusenko and Segrè 21] proposed a very interesting mechanism that the pulsar kicks may be induced by neutrino oscillation. Unfortunately as we have already noted above, this mechanism does not work unless we introduce very heavy sterile neutrinos, because with the experimentally inferred parameters neutrino oscillation is strongly suppressed near the neutrinosphere.

The remainder of this paper is organized as follows. In Sec. III, we derive a general formula for the matrix element and the cross section of the $\nu-N$ scatterings by considering the weakmagnetism and recoil corrections. A more concrete form of the cross section is derived in Sec. III based on the reasonable assumption that the nucleons are highly nondegenerate. In Sec. IV using a simple diffusion approximation, the drift flux due to $\nu$ - $N$ scatterings is given in a very simple form, and we discuss the implications of the diffusion equation in Sec. D. 


\section{MATRIX ELEMENT AND DIFFERENTIAL CROSS SECTION}

We consider neutrino-nucleon scatterings via the neutral-current interaction in magnetic fields. The usual $V-A$ current is modified when the weak magnetism correction is included as

$$
c_{V} \gamma^{\mu}+F_{2} \frac{i \sigma^{\mu \nu} q_{\nu}}{2 m}-c_{A} \gamma^{\mu} \gamma^{5}
$$

where $q^{\mu}=k^{\mu}-\left(k^{\mu}\right)^{\prime}$ is the momentum transfered to the nucleon. We label the neutrino momentum by $k$, and the nucleon momentum by $p$; for the final state momenta, the symbol $'$ is attached. We summarize the values of couplings, $c_{V}, c_{A}$, and $F_{2}$ in Table $\llbracket$.

TABLE I: Coupling constants. Here $g_{A} \approx 1.26, \sin ^{2} \theta_{W} \approx 0.23, \mu_{p}=1.793$, and $\mu_{n}=-1.913$.

\begin{tabular}{lccc}
\hline \hline Reaction & $c_{V}$ & $c_{A}$ & $F_{2}$ \\
\hline$\nu p \rightarrow \nu p$ & $\frac{1}{2}-2 \sin ^{2} \theta_{W}$ & $\frac{g_{A}}{2}$ & $\frac{1}{2}\left(\mu_{p}-\mu_{n}\right)-2 \mu_{p} \sin ^{2} \theta_{W}$ \\
$\nu n \rightarrow \nu n$ & $-\frac{1}{2}$ & $-\frac{g_{A}}{2}-\frac{1}{2}\left(\mu_{p}-\mu_{n}\right)-2 \mu_{n} \sin ^{2} \theta_{W}$ \\
$\nu_{e} n \rightarrow e^{-} p$ & 1 & $g_{A}$ & $\mu_{p}-\mu_{n}$ \\
$\bar{\nu}_{e} p \rightarrow e^{+} n$ & 1 & $g_{A}$ & $\mu_{p}-\mu_{n}$ \\
\hline \hline
\end{tabular}

Since the weak magnetism correction is at $k / m$ level, we must also include the nucleon recoil correction of the same order. The initial and final states of nucleon spinor can then be expressed by

$$
\begin{aligned}
u_{N}^{s}(p) \bar{u}_{N}^{s}(p) & =\frac{1}{2}\left[\left(1-s \gamma^{5} \gamma^{3}\right)\left(1+\gamma^{0}+\frac{p_{i}}{m} \gamma^{i}\right)-s \frac{p_{3}}{m} \gamma^{5}\left(1+\gamma^{0}\right)\right], \\
u_{N}^{s^{\prime}}\left(p^{\prime}\right) \bar{u}_{N}^{s^{\prime}}\left(p^{\prime}\right) & =\frac{1}{2}\left[\left(1-s^{\prime} \gamma^{5} \gamma^{3}\right)\left(1+\gamma^{0}+\frac{p_{i}^{\prime}}{m} \gamma^{i}\right)-s^{\prime} \frac{p_{3}^{\prime}}{m} \gamma^{5}\left(1+\gamma^{0}\right)\right],
\end{aligned}
$$

where $s, s^{\prime}= \pm 1$ specify the initial and final nucleon spins relative to the magnetic field axis (3-axis).

With these corrections, the matrix element for $\nu$ - $N$ scattering is given, to the order of $k / m$, by

$$
\left|\mathcal{M}_{s s^{\prime}}\left(\boldsymbol{\Omega}, \boldsymbol{\Omega}^{\prime}, \boldsymbol{p}, \boldsymbol{p}^{\prime}\right)\right|^{2}=\left|\mathcal{M}_{s s^{\prime}}^{(0)}\left(\boldsymbol{\Omega}, \boldsymbol{\Omega}^{\prime}\right)\right|^{2}+\delta\left|\mathcal{M}_{s s^{\prime}}\left(\boldsymbol{\Omega}, \boldsymbol{\Omega}^{\prime}, \boldsymbol{q}\right)\right|^{2}+\delta\left|\tilde{\mathcal{M}}_{s s^{\prime}}\left(\boldsymbol{\Omega}, \boldsymbol{\Omega}^{\prime}, \boldsymbol{P}\right)\right|^{2},
$$

where $\boldsymbol{\Omega}=\boldsymbol{k} / k$ and $\boldsymbol{\Omega}^{\prime}=\boldsymbol{k}^{\prime} / k^{\prime} .\left|\mathcal{M}_{s s^{\prime}}^{(0)}\left(\boldsymbol{\Omega}, \boldsymbol{\Omega}^{\prime}\right)\right|^{2}$ is the leading term already given in Ref. 14] by,

$$
\left|\mathcal{M}_{s s^{\prime}}^{(0)}\left(\boldsymbol{\Omega}, \boldsymbol{\Omega}^{\prime}\right)\right|^{2}=\frac{G_{F}^{2}}{2}\left[c_{V}^{2}+3 c_{A}^{2}+\left(c_{V}^{2}-c_{A}^{2}\right) \boldsymbol{\Omega} \cdot \boldsymbol{\Omega}^{\prime}+2 c_{A}\left(c_{V}+c_{A}\right)\left(s \boldsymbol{\Omega}+s^{\prime} \boldsymbol{\Omega}^{\prime}\right) \cdot \hat{\boldsymbol{B}}\right.
$$




$$
\begin{aligned}
& +2 c_{A}\left(c_{V}-c_{A}\right)\left(s \boldsymbol{\Omega}^{\prime}+s^{\prime} \boldsymbol{\Omega}\right) \cdot \hat{\boldsymbol{B}}+s s^{\prime}\left\{\left(c_{V}^{2}-c_{A}^{2}\right)\left(1+\boldsymbol{\Omega} \cdot \boldsymbol{\Omega}^{\prime}\right)\right. \\
& \left.\left.+4 c_{A}^{2} \boldsymbol{\Omega} \cdot \hat{\boldsymbol{B}} \boldsymbol{\Omega}^{\prime} \cdot \hat{\boldsymbol{B}}\right\}\right]
\end{aligned}
$$

$\delta\left|\mathcal{M}_{s s^{\prime}}\left(\boldsymbol{\Omega}, \boldsymbol{\Omega}^{\prime}, \boldsymbol{q}\right)\right|^{2}$ is the correction term at $\mathcal{O}(k / m)$ which depends on $\boldsymbol{q}\left(=\boldsymbol{p}^{\prime}-\boldsymbol{p}=\boldsymbol{k}-\boldsymbol{k}^{\prime}\right)$ as well as $\Omega$ and $\boldsymbol{\Omega}^{\prime}$ :

$$
\begin{aligned}
\delta\left|\mathcal{M}_{s s^{\prime}}\left(\boldsymbol{\Omega}, \boldsymbol{\Omega}^{\prime}, \boldsymbol{q}\right)\right|^{2}= & \frac{G_{F}^{2}}{2 m}\left[ \pm 2 c_{A}\left(c_{V}+F_{2}\right) \boldsymbol{q} \cdot\left(\boldsymbol{\Omega}-\boldsymbol{\Omega}^{\prime}\right)+2 c_{A}\left(c_{V}+F_{2}\right)\left(s-s^{\prime}\right) \boldsymbol{q} \cdot \hat{\boldsymbol{B}}\right. \\
& \pm 2 c_{A}\left(c_{V}+F_{2}\right) s s^{\prime} \boldsymbol{q} \cdot \hat{\boldsymbol{B}}\left(\boldsymbol{\Omega}^{\prime}-\boldsymbol{\Omega}\right) \cdot \hat{\boldsymbol{B}} \\
& +c_{A}\left(c_{V}+F_{2}\right)\left(s^{\prime}-s\right)\left(\boldsymbol{q} \cdot \boldsymbol{\Omega} \boldsymbol{\Omega}^{\prime} \cdot \hat{\boldsymbol{B}}+\boldsymbol{q} \cdot \boldsymbol{\Omega}^{\prime} \boldsymbol{\Omega} \cdot \hat{\boldsymbol{B}}\right) \\
& \left.\mp c_{V}\left(c_{V}+F_{2}\right)\left(s+s^{\prime}\right)\left\{\left(\boldsymbol{\Omega}^{\prime} \times \boldsymbol{\Omega}\right) \times \boldsymbol{q}\right\} \cdot \hat{\boldsymbol{B}}\right],
\end{aligned}
$$

and $\delta\left|\tilde{\mathcal{M}}_{s s^{\prime}}\left(\boldsymbol{\Omega}, \boldsymbol{\Omega}^{\prime}, \boldsymbol{P}\right)\right|^{2}$ is also the correction term at $\mathcal{O}(k / m)$, which depends on $\boldsymbol{P}\left(\equiv \boldsymbol{p}+\boldsymbol{p}^{\prime}\right)$ :

$$
\begin{aligned}
\delta\left|\tilde{\mathcal{M}}_{s s^{\prime}}\left(\boldsymbol{\Omega}, \boldsymbol{\Omega}^{\prime}, \boldsymbol{P}\right)\right|^{2}= & \frac{G_{F}^{2}}{2 m}\left[-\left\{c_{V}^{2}+c_{A}^{2}+\left(c_{V}^{2}-c_{A}^{2}\right) s s^{\prime}\right\} \boldsymbol{P} \cdot\left(\boldsymbol{\Omega}+\boldsymbol{\Omega}^{\prime}\right)-2 c_{V} c_{A}\left(s+s^{\prime}\right) \boldsymbol{P} \cdot \hat{\boldsymbol{B}}\right. \\
& -2 c_{A}^{2} s s^{\prime} \boldsymbol{P} \cdot \hat{\boldsymbol{B}}\left(\boldsymbol{\Omega}+\boldsymbol{\Omega}^{\prime}\right) \cdot \hat{\boldsymbol{B}}-c_{V} c_{A}\left(s+s^{\prime}\right)\left(\boldsymbol{P} \cdot \boldsymbol{\Omega} \boldsymbol{\Omega}^{\prime} \cdot \hat{\boldsymbol{B}}\right. \\
& \left.\left.+\boldsymbol{P} \cdot \boldsymbol{\Omega}^{\prime} \boldsymbol{\Omega} \cdot \hat{\boldsymbol{B}}\right) \pm c_{A}\left(s^{\prime}-s\right)\left\{\left(\boldsymbol{\Omega}^{\prime} \times \boldsymbol{\Omega}\right) \times \boldsymbol{P}\right\} \cdot \hat{\boldsymbol{B}}\right] .
\end{aligned}
$$

Here, upper and lower signs represent the expressions for neutrinos and antineutrinos, respectively; this notation is kept throughout this paper. Note that we can explicitly check the time-reversal symmetry for the matrix element $\left|\mathcal{M}_{s s^{\prime}}\left(\Omega, \Omega^{\prime}, \boldsymbol{p}, \boldsymbol{p}^{\prime}\right)\right|^{2}$, by exchanging all the initial and final state quantities, i.e., $\boldsymbol{\Omega} \leftrightarrow \boldsymbol{\Omega}^{\prime}, \boldsymbol{P} \rightarrow \boldsymbol{P}, \boldsymbol{q} \rightarrow-\boldsymbol{q}$, and $s \leftrightarrow s^{\prime}$.

The differential cross section (per unit volume) can be obtained from the matrix element through the expression,

$$
\begin{aligned}
\frac{d \Gamma}{d k^{\prime} d \Omega^{\prime}}= & \frac{k^{\prime 2}}{(2 \pi)^{3}} \sum_{s s^{\prime}} \int \frac{d^{3} p}{(2 \pi)^{3}} \frac{d^{3} p^{\prime}}{(2 \pi)^{3}}(2 \pi)^{4} \delta^{4}\left(p+k-p^{\prime}-k^{\prime}\right) f_{N}\left(1-f_{N}^{\prime}\right) \\
& \times\left|\mathcal{M}_{s s^{\prime}}\left(\boldsymbol{\Omega}, \boldsymbol{\Omega}^{\prime}, \boldsymbol{p}, \boldsymbol{p}^{\prime}\right)\right|^{2}
\end{aligned}
$$

where $f_{N} \equiv f_{N}(E), f_{N}^{\prime} \equiv f_{N}\left(E^{\prime}\right), f_{N}(E)$ is the nucleon distribution function with energy $E$ given by

$$
f_{N}(E)=\frac{1}{\exp \left[\left(E-\mu_{N}\right) / T\right]+1}
$$

and $\mu_{N}$ is the nucleon chemical potential (excluding rest mass). In evaluating the momentum integral, we neglect the Landau levels of nucleons, and therefore the nucleon momentum is a well-defined quantity. This is justified because many Landau levels are occupied for the conditions in a protoneutron star, and the change in the available phase space due to the 
Landau levels is negligible. By substituting Eqs. (44)-(6) into Eq. (7), the $\nu$ - $N$ cross section in the magnetic fields can be calculated to the order of $k / m$. Equation (77) is written in more convenient form as

$$
\begin{aligned}
\frac{d \Gamma}{d k^{\prime} d \Omega^{\prime}}= & A_{0}\left(k, k^{\prime}, \mu^{\prime}\right)+\delta A_{ \pm}\left(k, k^{\prime}, \mu^{\prime}\right) \boldsymbol{\Omega} \cdot \hat{\boldsymbol{B}}+\delta A_{\mp}\left(k, k^{\prime}, \mu^{\prime}\right) \boldsymbol{\Omega}^{\prime} \cdot \hat{\boldsymbol{B}} \\
& +A_{0}^{W M}\left(k, k^{\prime}, \mu^{\prime}\right)+\delta B^{W M}\left(k, k^{\prime}, \mu^{\prime}\right) \boldsymbol{\Omega} \cdot \hat{\boldsymbol{B}}+\delta C^{W M}\left(k, k^{\prime}, \mu^{\prime}\right) \boldsymbol{\Omega}^{\prime} \cdot \hat{\boldsymbol{B}}
\end{aligned}
$$

where $\mu^{\prime}=\Omega \cdot \Omega^{\prime} . \quad A_{0}$ and $\delta A_{ \pm}$are the terms which include the matrix element $\left|\mathcal{M}_{s s^{\prime}}^{(0)}\left(\Omega, \Omega^{\prime}\right)\right|^{2}:$

$$
\begin{aligned}
A_{0}\left(k, k^{\prime}, \mu^{\prime}\right) & =\frac{k^{\prime 2}}{(2 \pi)^{3}} \sum_{s, s^{\prime}}\left|\mathcal{M}_{s s^{\prime}}^{(0)}\left(\boldsymbol{\Omega}, \boldsymbol{\Omega}^{\prime}\right)\right|^{2} S_{0}\left(q_{0}, q\right), \\
\delta A_{ \pm} \boldsymbol{\Omega} \cdot \hat{\boldsymbol{B}}+\delta A_{\mp} \boldsymbol{\Omega}^{\prime} \cdot \hat{\boldsymbol{B}} & =\frac{k^{\prime 2}}{(2 \pi)^{3}} \sum_{s, s^{\prime}}\left|\mathcal{M}_{s s^{\prime}}^{(0)}\left(\boldsymbol{\Omega}, \boldsymbol{\Omega}^{\prime}\right)\right|^{2} \delta S_{s s^{\prime}}\left(q_{0}, q\right),
\end{aligned}
$$

where $S_{0}\left(q_{0}, q\right)$ and $\delta S_{s s^{\prime}}\left(q_{0}, q\right)$ can be derived from the "nuclear response function," $S_{s s^{\prime}}\left(q_{0}, q\right)=S_{0}\left(q_{0}, q\right)+\delta S_{s s^{\prime}}\left(q_{0}, q\right)$, defined by

$$
S_{s s^{\prime}}\left(q_{0}, q\right)=\int \frac{d^{3} p}{(2 \pi)^{3}} \frac{d^{3} p^{\prime}}{(2 \pi)^{3}}(2 \pi)^{4} \delta^{4}\left(p+k-p^{\prime}-k^{\prime}\right) f_{N}\left(1-f_{N}^{\prime}\right) .
$$

$S_{0}\left(q_{0}, q\right)$ is the leading term when $B=0$, and $\delta S_{s s^{\prime}}\left(q_{0}, q\right)$ is the correction arising from nonzero $B$, which can be simplified, for small $B$ compared to temperature $\left(\mu_{B} B / T \ll 1\right)$, to be

$$
\begin{aligned}
S_{0}\left(q_{0}, q\right) & =\frac{m^{2} T}{2 \pi q} \frac{1}{1-e^{-z}} \ln \left(\frac{1+e^{-x_{0}}}{1+e^{-x_{0}-z}}\right), \\
\delta S_{s s^{\prime}} & =-\frac{m^{2} T}{2 \pi q} \frac{\delta x}{\left(e^{x_{0}}+1\right)\left(1+e^{-x_{0}-z}\right)},
\end{aligned}
$$

with the parameters given by

$$
\begin{aligned}
z & =\frac{q_{0}}{T}, \quad x_{0}=\frac{\left(q_{0}-q^{2} / 2 m\right)^{2}}{4 T\left(q^{2} / 2 m\right)}-\frac{\mu_{N}}{T} \\
\delta x & =-\frac{\mu_{B} B}{2 T}\left[\left(1+\frac{2 m q_{0}}{q^{2}}\right) s+\left(1-\frac{2 m q_{0}}{q^{2}} s^{\prime}\right)\right] .
\end{aligned}
$$

The detailed derivations of these relations have been already discussed in Ref. [14], and we refer the reader to the literature. The terms including $A_{0}^{W M}, \delta B^{W M}$, and $\delta C^{W M}$, which are smaller than $A_{0}$ and $\delta A_{ \pm}$by factor $k / m$, are calculated by

$$
A_{0}^{W M}+\delta B^{W M} \boldsymbol{\Omega} \cdot \hat{\boldsymbol{B}}+\delta C^{W M} \boldsymbol{\Omega}^{\prime} \cdot \hat{\boldsymbol{B}}
$$




$$
\begin{aligned}
= & \frac{k^{\prime 2}}{(2 \pi)^{3}} \sum_{s, s^{\prime}}\left[\delta\left|\mathcal{M}_{s s^{\prime}}\left(\boldsymbol{\Omega}, \boldsymbol{\Omega}^{\prime}, \boldsymbol{q}\right)\right|^{2}\left\{S_{0}\left(q_{0}, q\right)+\delta S_{s s^{\prime}}\left(q_{0}, q\right)\right\}\right. \\
& \left.+\int \frac{d^{3} p}{(2 \pi)^{3}} \frac{d^{3} p^{\prime}}{(2 \pi)^{3}}(2 \pi)^{4} \delta^{4}\left(p+k-p^{\prime}-k^{\prime}\right) f_{N}\left(1-f_{N}^{\prime}\right) \delta\left|\tilde{\mathcal{M}}_{s s^{\prime}}\left(\boldsymbol{\Omega}, \boldsymbol{\Omega}^{\prime}, \boldsymbol{P}\right)\right|^{2}\right]
\end{aligned}
$$

where in the second term, we cannot take the matrix element out of the integral because it depends on $\boldsymbol{p}$ and $\boldsymbol{p}^{\prime}$, unlike that in the first term which depends on $\boldsymbol{q}=\boldsymbol{k}-\boldsymbol{k}^{\prime}$.

Equations (10), (11), and (16) are quite difficult to evaluate at this stage. However, we can calculate it assuming that the nucleons are nondegenerate, as we show in the next section. In fact, this assumption appears to be appropriate, because the asymmetric drift flux can develop only when the neutrino distribution deviates from thermal equilibrium; this occurs in the regime where nucleons are nondegenerate.

\section{NONDEGENERATE NUCLEON LIMIT}

For nondegenerate nucleons, the characteristic neutrino energy transfer in each scattering is of order $q_{0} \sim k(T / m)^{1 / 2} \ll k$. The cross section sharply peaks around $k^{\prime}=k$, and we can evaluate $A_{0}, \delta A_{ \pm}, A_{0}^{W M}, \delta B^{W M}$, and $\delta C^{W M}$, in a series of the small parameter $(T / m)^{1 / 2}$. We define the dimensionless quantities

$$
\epsilon=\left[\frac{4\left(1-\mu^{\prime}\right) T}{m}\right]^{1 / 2}, u=\frac{k^{\prime}-k}{\epsilon k},
$$

where the range of $u$ is from $-1 / \epsilon$ to $\infty$. Using the expansions of the momentum integrals given in Appendix A, we obtain the following expressions,

$$
\begin{aligned}
S_{0}\left(q_{0}, q\right)= & \frac{m^{2} T}{2 \pi q} n\left(\frac{2 \pi^{3}}{m^{3} T^{3}}\right)^{\frac{1}{2}} \exp \left[-\frac{\left(q_{0}-q^{2} / 2 m\right)^{2}}{4 T q^{2} /(2 m)}\right] \\
\simeq & \frac{\pi^{1 / 2} n}{\epsilon k} e^{-u^{2}}\left[1-\frac{1}{2}\left(1+\frac{k}{T}\right) \epsilon u+\epsilon u^{3}+\mathcal{O}\left(\epsilon^{2}\right)\right] \\
A_{0}= & \frac{k^{\prime 2}}{(2 \pi)^{3}} 2 G_{F}^{2}\left[c_{V}^{2}+3 c_{A}^{2}+\left(c_{V}^{2}-c_{A}^{2}\right) \mu^{\prime}\right] S_{0}\left(q_{0}, q\right), \\
\delta A_{ \pm}= & \frac{k^{\prime 2}}{(2 \pi)^{3}} 4 G_{F}^{2} c_{A} \frac{\mu_{B} B}{T}\left(c_{V} \pm 2 c_{A} \frac{m q_{0}}{q^{2}}\right) S_{0}\left(q_{0}, q\right), \\
A_{0}^{W M}= & -\frac{k^{\prime 2}}{(2 \pi)^{3}} \frac{4 G_{F}^{2}}{m}\left[\left(c_{V}^{2}+c_{A}^{2}\right) \frac{m q_{0}}{q^{2}} \boldsymbol{q} \cdot\left(\boldsymbol{\Omega}+\boldsymbol{\Omega}^{\prime}\right) \pm c_{A}\left(c_{V}+F_{2}\right) \boldsymbol{q} \cdot\left(\boldsymbol{\Omega}^{\prime}-\boldsymbol{\Omega}\right)\right] \\
\delta B^{W M}= & \frac{k^{\prime 2}}{(2 \pi)^{3}} \frac{2 G_{F}^{2}}{m} \frac{\mu_{B} B}{T}\left[4 c_{A} F_{2} \frac{m q_{0}}{q^{2}} k-2 c_{A}\left(2 c_{V}+F_{2}\right) \frac{m q_{0}}{q^{2}} \boldsymbol{q} \cdot \boldsymbol{\Omega}^{\prime}\right.
\end{aligned}
$$




$$
\begin{aligned}
& \left. \pm\left\{4 c_{A}^{2} \frac{m T}{q^{2}}\left(1-\frac{m q_{0}^{2}}{T q^{2}}\right)-c_{V}\left(c_{V}+F_{2}\right)\right\} \boldsymbol{q} \cdot \boldsymbol{\Omega}^{\prime}\right] S_{0}\left(q_{0}, q\right), \\
\delta C^{W M}= & \frac{k^{\prime 2}}{(2 \pi)^{3}} \frac{2 G_{F}^{2}}{m} \frac{\mu_{B} B}{T}\left[-4 c_{A} F_{2} \frac{m q_{0}}{q^{2}} k^{\prime}-2 c_{A}\left(2 c_{V}+F_{2}\right) \frac{m q_{0}}{q^{2}} \boldsymbol{q} \cdot \boldsymbol{\Omega}\right. \\
& \left.\mp\left\{4 c_{A}^{2} \frac{m T}{q^{2}}\left(1-\frac{m q_{0}^{2}}{T q^{2}}\right)-c_{V}\left(c_{V}+F_{2}\right)\right\} \boldsymbol{q} \cdot \boldsymbol{\Omega}\right] S_{0}\left(q_{0}, q\right),
\end{aligned}
$$

where all of them are expressed by $u$ and $\epsilon$ instead of $q_{0}$ and $q$, through the relations such as $q_{0}=k-k^{\prime}=-\epsilon u k$ and $\boldsymbol{q}=\boldsymbol{k}-\boldsymbol{k}^{\prime}=k \boldsymbol{\Omega}-k(1+\epsilon u) \boldsymbol{\Omega}^{\prime}$. In deriving the expressions above, we have used the equation $e^{\mu_{N} / T}=n\left(2 \pi^{3} / m^{3} T^{3}\right)^{1 / 2}$ that is valid when $B=0$, to relate the nucleon chemical potential $\mu_{N}$ to its number density $n$ (the corrections due to finite $B$ are of order $B^{2}$ ). These expressions are valid under the conditions $T \ll m, k \ll(m T)^{1 / 2}$, $\mu_{B} B \ll T$, and $k \gtrsim k_{\min }=\mu_{B} B(m / T)^{1 / 2} \simeq 10^{-2}|g| B_{14} T^{-1 / 2} \mathrm{MeV}$. All these conditions are satisfied for the case of interest in our study. Unlike the previous publication [14], we must keep in the bracket of $S_{0}$ to $\mathcal{O}\left(\epsilon^{4}\right)$ terms although we did not explicitly show them in Eq. (18). This is because they are necessary for the evaluation of the drift flux which is suppressed at $\mathcal{O}(k / m)$ level compared with the leading term.

Thus, we have derived the useful form of the differential cross section of neutrino-nucleon scatterings, which enables us to discuss the parity violating effect of the neutral-current weak interaction related to pulsar kicks. At first sight, however, these equations are still too complicated to obtain an intuitive implication for the asymmetric neutrino emission in the supernova magnetic field. Therefore, in the remainder of this paper, we derive a simple diffusion equation with which we can see how large drift flux is obtained. More delicate treatment using numerical simulations is beyond the scope of this study; it is slated for the future work.

\section{DIFFUSION EQUATION FOR A DRIFT FLUX}

The Boltzmann equation for neutrino transport is written in the form

$$
\frac{\partial f_{\nu}(\boldsymbol{k})}{\partial t}+\boldsymbol{\Omega} \cdot \nabla f_{\nu}(\boldsymbol{k})=\left[\frac{\partial f_{\nu}(\boldsymbol{k})}{\partial t}\right]_{\mathrm{sc}},
$$

where we include the collision term due to scattering alone; effects of absorption are not considered in this section. The collision term in Eq. (24) can be written by the following 
integral [14]:

$$
\left[\frac{\partial f_{\nu}(\boldsymbol{k})}{\partial t}\right]_{\mathrm{sc}}=\int_{0}^{\infty} d k^{\prime} \int d \Omega^{\prime} \frac{d \Gamma}{d k^{\prime} d \Omega^{\prime}}\left[C\left(k, k^{\prime}\right) \delta f_{\nu}^{\prime}+D\left(k, k^{\prime}\right) \delta f_{\nu}\right]
$$

where $\delta f_{\nu}$ and $\delta f_{\nu}^{\prime}$ denote the deviation of neutrino distribution function from the value of equilibrium, i.e., $\delta f_{\nu}=f_{\nu}-f_{\nu}^{(0)}$. The coefficients of $\delta f_{\nu}$ and $\delta f_{\nu}^{\prime}$ are

$$
\begin{aligned}
& C\left(k, k^{\prime}\right)=e^{-q_{0} / T}\left(1-f_{\nu}^{(0)}\right)+f_{\nu}^{(0)} \\
& D\left(k, k^{\prime}\right)=-\left[e^{-q_{0} / T} f_{\nu}^{(0) \prime}+1-f_{\nu}^{(0) \prime}\right] .
\end{aligned}
$$

The nonlinear terms which are proportional to $\delta f_{\nu} \delta f_{\nu}^{\prime}$ were dropped since we consider the regime where the deviation from thermal equilibrium $\delta f_{\nu}$ is relatively small (the regime where the diffusion approximation is valid).

For the purpose of examining the macroscopic consequence of the asymmetric cross section, we expand the neutrino distribution function in spherical harmonics up to dipole order as

$$
\delta f_{\nu}(\boldsymbol{k})=g(k)+3 \boldsymbol{\Omega} \cdot \boldsymbol{h}(k)
$$

where $g(k)$ is the spherically symmetric deviation from the equilibrium, and $\boldsymbol{h}(k)$ represents the dipole deviation which leads to the flux. These quantities, $g(k)$ and $\boldsymbol{h}(k)$ are related to the energy density and the flux through the relations

$$
\begin{aligned}
U_{\nu}(k) & =\int \frac{k^{2} d \Omega}{(2 \pi)^{3}} k f_{\nu}=\frac{k^{3}}{2 \pi^{2}}\left[f_{\nu}^{(0)}(k)+g(k)\right]=U_{\nu}^{\mathrm{FD}}(k)+\frac{k^{3}}{2 \pi^{2}} g(k), \\
\boldsymbol{F}_{\nu}(k) & =\int \frac{k^{2} d \Omega}{(2 \pi)^{3}} k \Omega f_{\nu}=\frac{k^{3}}{2 \pi^{2}} \boldsymbol{h}(k),
\end{aligned}
$$

where the quantity with superscript FD represents the value in the case of thermal equilibrium.

The first moment of Eq. (25) yields

$$
\int \frac{d \Omega}{4 \pi} \boldsymbol{\Omega}\left[\frac{\partial f_{\nu}(\boldsymbol{k})}{\partial t}\right]_{\mathrm{sc}}=\boldsymbol{V}_{0} \pm \delta \boldsymbol{V}_{ \pm}^{B}+\delta \boldsymbol{V}_{0}^{W M} \pm \delta \boldsymbol{V}_{ \pm}^{W M}+\delta \boldsymbol{V}_{0}^{B, W M} \pm \delta \boldsymbol{V}_{ \pm}^{B, W M}
$$

where the subscript "0" of $\boldsymbol{V}$ and $\delta \boldsymbol{V}$ represents that the terms are the same for neutrinos and antineutrinos, while " \pm " that the sign of each term is different for neutrinos and antineutrinos. The term $\boldsymbol{V}_{0}$ contributes at the leading level, whereas the quantities with superscript " $B$," "WM," and " $B, W M$ " are suppressed by factors of $\mathcal{O}\left(\mu_{B} B / T\right), \mathcal{O}(T / m)$, 
and $\mathcal{O}\left(\mu_{B} B / m\right)$, respectively. As a result of lengthy calculations (details are given in Ref. [14]), each term is written as

$$
\begin{aligned}
\boldsymbol{V}_{0}= & -\frac{2 G_{F}^{2} k^{2}}{3 \pi} n\left(c_{V}^{2}+5 c_{A}^{2}\right) \boldsymbol{h}, \\
\delta \boldsymbol{V}_{ \pm}^{B}= & -\frac{4 G_{F}^{2} k^{2}}{3 \pi} n \frac{\mu_{B} B}{T} c_{A}^{2}\left[\left(1-2 f_{\nu}^{(0)}\right) g+T \frac{\partial g}{\partial k}\right] \hat{\boldsymbol{B}} \\
\delta \boldsymbol{V}_{0}^{W M}= & -\frac{2 G_{F}^{2} k^{2}}{3 \pi} n \frac{k}{m}\left[\left\{-3\left(c_{V}^{2}+3 c_{A}^{2}\right)\left(1-2 f_{\nu}^{(0)}\right)+5\left(c_{V}^{2}+9 c_{A}^{2}\right) \frac{T}{k}\right.\right. \\
& \left.\left.+2\left(c_{V}^{2}+5 c_{A}^{2}\right) k \frac{\partial f_{\nu}^{(0)}}{\partial k}\right\} \boldsymbol{h}+2 c_{A}^{2}\left(1-2 f_{\nu}^{(0)}+\frac{6 T}{k}\right) k \frac{\partial \boldsymbol{h}}{\partial k}+2 c_{A}^{2} k T \frac{\partial^{2} \boldsymbol{h}}{\partial k^{2}}\right], \\
\delta \boldsymbol{V}_{ \pm}^{W M}= & -\frac{16 G_{F}^{2} k^{2}}{3 \pi} n \frac{k}{m} c_{A}\left(c_{V}+F_{2}\right) \boldsymbol{h}, \\
\delta \boldsymbol{V}_{0}^{B, W M}= & -\frac{4 G_{F}^{2} k^{2}}{9 \pi} n \frac{\mu_{B} B}{T} \frac{k}{m} c_{A}\left[\left\{\left(-2 c_{V}+5 F_{2}\right)\left(1-2 f_{\nu}^{(0)}\right)+2 c_{V} k \frac{\partial f_{\nu}^{(0)}}{\partial k}\right\} g\right. \\
& \left.-\left\{c_{V}\left(1-2 f_{\nu}^{(0)}\right)+\left(2 c_{V}-5 F_{2}\right) \frac{T}{k}\right\} k \frac{\partial g}{\partial k}-c_{V} k T \frac{\partial^{2} g}{\partial k^{2}}\right] \hat{\boldsymbol{B}}, \\
\delta \boldsymbol{V}_{ \pm}^{B, W M}= & -\frac{4 G_{F}^{2} k^{2}}{9 \pi} n \frac{\mu_{B} B}{m} c_{A}^{2}\left[\left\{69\left(1-2 f_{\nu}^{(0)}\right)-68 k \frac{\partial f_{\nu}^{(0)}}{\partial k}\right.\right. \\
& \left.-12 k^{2} \frac{\partial^{2} f_{\nu}^{(0)}}{\partial k^{2}}\right\} g+\left\{34\left(1-2 f_{\nu}^{(0)}\right)+\frac{2 k}{T}+\frac{69 T}{k}\right\} k \frac{\partial g}{\partial k} \\
& \left.+2\left\{3\left(1-2 f_{\nu}^{(0)}\right)+\frac{17 T}{k}\right\} k^{2} \frac{\partial^{2} g}{\partial k^{2}}+4 k^{2} T \frac{\partial^{3} g}{\partial k^{3}}\right] \hat{\boldsymbol{B} .} \\
& +3{ }^{2},
\end{aligned}
$$

Since the sign of the $\mathcal{O}\left(\mu_{B} B / T\right)$ term is opposite for neutrinos and antineutrinos, there occurs cancellation of the drift flux at this level. Hence, the leading drift flux due to $\nu-N$ scatterings is suppressed by a very small coefficient of $\mathcal{O}\left(\mu_{B} B / m\right)$.

When we discuss the terms which depend on quantities such as $\partial \boldsymbol{h} / \partial k, \partial^{2} \boldsymbol{h} / \partial k^{2}$, we use the lowest order expression for $\boldsymbol{h}$, i.e.,

$$
\begin{aligned}
\boldsymbol{h}(k) & \simeq-\frac{1}{3 \kappa_{0}} \nabla\left(f_{\nu}^{(0)}(k)+g(k)\right) \\
\kappa_{0} & =\frac{2 G_{F}^{2} k^{2}}{3 \pi} n\left(c_{V}^{2}+5 c_{A}^{2}\right)
\end{aligned}
$$

In this and the following expressions for the diffusion equations, we drop the time derivative term; it corresponds to a rapid redistribution of matter temperature, whose time scale is of order the mean free path divided by the speed of light $c$, much smaller than neutrino diffusion time of the star. In addition, we assume that all the quantities except for $\boldsymbol{h}$, such as $g, f_{\nu}^{(0)}$, are the same for neutrinos and antineutrinos. All these assumptions are considered 
to be quite applicable, because the discrepancy caused by them is further suppressed to the extent in which we are not interested. With the above assumptions, we take the first moment of the Boltzmann equation (24), and obtain the explicit expression for the neutrino plus antineutrino flux $\boldsymbol{F}_{\nu}+\boldsymbol{F}_{\bar{\nu}}$ :

$$
\begin{aligned}
\boldsymbol{F}_{\nu}(k)+\boldsymbol{F}_{\bar{\nu}}(k)= & -\frac{2}{3 \kappa_{0}}\left(1-\delta^{(1)}\right) \nabla U_{\nu}(k) \\
& +\delta^{(2)}\left[\left(1-2 f_{\nu}^{(0)}+\frac{6 T}{k}\right) k \frac{\partial}{\partial k}\left(\frac{\nabla U_{\nu}(k)}{\kappa_{0}}\right)+k T \frac{\partial^{2}}{\partial k^{2}}\left(\frac{\nabla U_{\nu}(k)}{\kappa_{0}}\right)\right] \\
& +\left[\epsilon^{(1)} \delta U_{\nu}(k)+\epsilon^{(2)} k \frac{\partial}{\partial k} \delta U_{\nu}(k)+\epsilon^{(3)} k^{2} \frac{\partial^{2}}{\partial k^{2}} \delta U_{\nu}(k)\right] \hat{\boldsymbol{B}}
\end{aligned}
$$

where $\delta^{(1)}$ and $\delta^{(2)}$ are small numbers of $\mathcal{O}(k / m)$, and $\epsilon^{(1)}, \epsilon^{(2)}$, and $\epsilon^{(3)}$ are those of $\mathcal{O}\left(\mu_{B} B / m\right)$; they are given in concrete forms by

$$
\begin{aligned}
\delta^{(1)}= & \frac{k}{m} \frac{1}{c_{V}^{2}+5 c_{A}^{2}}\left[-3\left(c_{V}^{2}+3 c_{A}^{2}\right)\left(1-2 f_{\nu}^{(0)}\right)-5\left(c_{V}^{2}+9 c_{A}^{2}\right) \frac{T}{k}\right. \\
& \left.+2\left(c_{V}^{2}+5 c_{A}^{2}\right) k \frac{\partial f_{\nu}^{(0)}}{\partial k}\right], \\
\delta^{(2)}= & \frac{4 k}{3 m} \frac{c_{A}^{2}}{c_{V}^{2}+5 c_{A}^{2}}, \\
\epsilon^{(1)}= & \frac{4 k}{3 m} \frac{\mu_{B} B}{T} \frac{c_{A}}{\left(c_{V}^{2}+5 c_{A}^{2}\right)^{2}}\left[\left\{2 c_{V}\left(c_{V}^{2}+17 c_{A}^{2}\right)-F_{2}\left(5 c_{V}^{2}+c_{A}^{2}\right)\right\}\left(1-2 f_{\nu}^{(0)}\right)\right. \\
& \left.-2 c_{V}\left(c_{V}^{2}+5 c_{A}^{2}\right) k \frac{\partial f_{\nu}^{(0)}}{\partial k}\right], \\
\epsilon^{(2)}= & \frac{4 k}{3 m} \frac{\mu_{B} B}{T} \frac{c_{A}}{\left(c_{V}^{2}+5 c_{A}^{2}\right)^{2}}\left[c_{V}^{3}\left(1+\frac{2 T}{k}\right)+c_{V} c_{A}^{2}\left(5+\frac{34 T}{k}\right)\right. \\
& \left.-2 c_{V}\left(c_{V}^{2}+5 c_{A}^{2}\right) f_{\nu}^{(0)}-F_{2}\left(5 c_{V}^{2}+c_{A}^{2}\right) \frac{T}{k}\right], \\
\epsilon^{(3)}= & \frac{4 \mu_{B} B}{3 m} \frac{c_{V} c_{A}}{c_{V}^{2}+5 c_{A}^{2}} .
\end{aligned}
$$

As expected and already pointed out by Arras and Lai [14], the drift flux, which is along the direction of the magnetic field $\boldsymbol{B}$, is absent at order $\mathcal{O}\left(\mu_{B} B / T\right)$; the leading term of the relevant drift flux is at $\mathcal{O}\left(\mu_{B} B / m\right)$ as shown in Eq. (40).

\section{DISCUSSION AND CONCLUSION}

Neutrinos carry away almost all the energy released by gravitational collapse of a supernova core, $\approx 3 \times 10^{53} \mathrm{erg}$, which is $\sim 100$ times the momentum associated with the spatial 
motion of pulsars. Therefore, $1 \%$ anisotropy in the momentum distribution of the outgoing neutrinos would suffice to account for the observed kick velocities. From Eq. (40), the kick due to $\nu$-N scatterings is characterized by the fractional momentum asymmetry factor

$$
\begin{aligned}
\alpha_{\mathrm{sc}} & =\frac{\int_{0}^{\infty} d k \int R_{\nu}^{2}(k) d \Omega\left[\boldsymbol{F}_{\nu}(k)+\boldsymbol{F}_{\bar{\nu}}(k)\right] \cdot \hat{\boldsymbol{B}}}{\int_{0}^{\infty} d k \int R_{\nu}^{2}(k) d \Omega\left[\boldsymbol{F}_{\nu}(k)+\boldsymbol{F}_{\bar{\nu}}(k)\right] \cdot \hat{\boldsymbol{r}}} \\
& \simeq \frac{\int_{0}^{\infty} d k R_{\nu}^{2}(k)\left[\epsilon^{(1)} \delta U_{\nu}(k)+\epsilon^{(2)} k \frac{\partial}{\partial k} \delta U_{\nu}(k)+\epsilon^{(3)} k^{2} \frac{\partial^{2}}{\partial k^{2}} \delta U_{\nu}(k)\right]}{\int_{0}^{\infty} d k R_{\nu}^{2}(k) \frac{2}{3 \kappa_{0}}\left|\frac{d U_{\nu}(k)}{d r}\right|_{\nu \text { sph. }}}
\end{aligned}
$$

where $R_{\nu}(k)$ represents a radius of the neutrinosphere of energy $k$. To evaluate the value of $\alpha_{\text {sc }}$ we must be careful, because the numerator of Eq. (46) contains $\delta U_{\nu}$ which is difficult to estimate without numerical simulations. However, we believe, for non-electron neutrinos and antineutrinos this deviation $\delta U_{\nu}$ can be large, which may cancel the small factors $\epsilon^{(i)}$. This is because of the fact as follows. The electron neutrinos and antineutrinos are kept in thermal equilibrium slightly within the neutrino sphere by beta processes, $\nu_{e} n \leftrightarrow e^{-} p$ and $\bar{\nu}_{e} p \leftrightarrow e^{+} n$; leading very little difference from thermal equilibrium $\delta U_{\nu_{e}, \bar{\nu}_{e}}$ at the neutrinosphere. In contrast, for $\nu_{\mu, \tau}$ and $\bar{\nu}_{\mu, \tau}$ the main source for the opacity is $\nu N \leftrightarrow \nu N$ reactions. However, since the nucleon mass is much larger than the relevant temperatures, energy exchange between neutrinos and nucleons is inefficient. Thus, the decoupling surface, within which neutrinos are kept in thermal equilibrium by the subdominant energy exchange processes such as $N N \leftrightarrow N N \nu \bar{\nu}, e^{+} e^{-} \leftrightarrow \nu \bar{\nu}$, and $\nu e \leftrightarrow \nu e$, locates at much deeper region than the neutrinosphere; it should lead rather large deviation $\delta U_{\nu}(k)$.

While a detailed study using numerical calculations is not given in the present paper, we simply compare our new drift term in Eq. (40) with the previously derived drift term due to absorption [14]. According to Arras and Lai [14], the drift flux due to the absorption $\nu_{e} n \rightarrow e^{-} p$ is described by

$$
\boldsymbol{F}_{\nu_{e}}^{\mathrm{drift}}=-\frac{\kappa_{0}^{*(\mathrm{abs})}}{3 \kappa_{0}^{(\mathrm{tot})}} \epsilon_{\mathrm{abs}} \delta U_{\nu_{e}}(k) \hat{\boldsymbol{B}},
$$

where $\kappa_{0}^{(\mathrm{tot})}=\kappa_{0}^{*(\mathrm{abs})}+\kappa_{0}, \kappa_{0}^{*(\mathrm{abs})}=\kappa_{0}^{(\mathrm{abs})}\left[1+e^{\left(\mu_{\nu}-k\right) / T}\right]$, with

$$
\begin{aligned}
\kappa_{0}^{(\mathrm{abs})}= & \frac{G_{F}^{2}}{\pi}\left(k+m_{n}-m_{p}\right)^{2} n_{n}\left(c_{V}^{2}+3 c_{A}^{2}\right)\left[1-f_{e}\left(k+m_{n}-m_{p}\right)\right], \\
\epsilon_{\mathrm{abs}}= & \frac{1}{2} \frac{e B}{\left(k+m_{n}-m_{p}\right)^{2}} \frac{c_{V}^{2}-c_{A}^{2}}{c_{V}^{2}+3 c_{A}^{2}}+2 \frac{c_{A}\left(c_{A}+c_{V}\right)}{c_{V}^{2}+3 c_{A}^{2}} \frac{\mu_{B n} B}{T} \\
& -\frac{T}{k+m_{n}-m_{p}}\left[1+\frac{k+m_{n}-m_{p}}{T} f_{e}\left(k+m_{n}-m_{p}\right)\right]
\end{aligned}
$$




$$
\times\left[2 \frac{c_{A}\left(c_{A}+c_{V}\right)}{c_{V}^{2}+3 c_{A}^{2}} \frac{\mu_{B n} B}{T}+2 \frac{c_{A}\left(c_{A}-c_{V}\right)}{c_{V}^{2}+3 c_{A}^{2}} \frac{\mu_{B p} B}{T}\right] .
$$

In order to compare the difference between the drift flux due to $\nu$ - $N$ scatterings and that due to absorption, we define the quantity $R_{\mathrm{sc} / \mathrm{abs}}$ by

$$
R_{\mathrm{sc} / \mathrm{abs}}=\left|\frac{2 \int_{0}^{\infty} d k\left[\epsilon^{(1)} \delta U_{\nu_{\mu}}(k)+\epsilon^{(2)} k \frac{\partial}{\partial k} \delta U_{\nu_{\mu}}(k)+\epsilon^{(3)} k^{2} \frac{\partial^{2}}{\partial k^{2}} \delta U_{\nu_{\mu}}(k)\right]}{\int_{0}^{\infty} d k \frac{\kappa_{0}^{*(\mathrm{abs})}}{3 \kappa_{0}^{(\mathrm{tot})}} \epsilon_{\mathrm{abs}} \delta U_{\nu_{e}}(k)}\right|,
$$

which is the ratio of the asymmetric fluxes due to the scattering and the absorption. The factor 2 in the numerator represents that the drift flux due to scatterings mainly comes from two-flavor $(\mu$ and $\tau$ ) neutrinos, whereas that due to absorptions is only related to electron neutrinos. More precisely, $R_{\nu}^{2}(k)$ must be included in the integral, but for simplicity we neglect it. In order to evaluate $R_{\mathrm{sc} / \mathrm{abs}}$, we need a further assumption concerning the energy densities $U_{\nu_{e}}$ and $U_{\nu_{\mu}}$. Here we parameterize them using the "effective temperature" $T_{e}^{\mathrm{eff}}$ and $T_{\mu}^{\mathrm{eff}}$ as follows:

$$
\begin{aligned}
U_{\nu_{e}}(k) & =\frac{k^{3}}{2 \pi^{2}} \frac{1}{e^{k / T_{e}^{\mathrm{eff}}}+1}, \\
U_{\nu_{\mu}}(k) & =\frac{k^{3}}{2 \pi^{2}} \frac{1}{e^{k / T_{\mu}^{\mathrm{eff}}}+1},
\end{aligned}
$$

where for further simplicity, $T_{e}^{\mathrm{eff}}$ and $T_{\mu}^{\mathrm{eff}}$ are assumed to be independent of $k$. As the other parameters at the neutrinosphere, we take $T=3 \mathrm{MeV}, \rho=10^{12} \mathrm{~g} \mathrm{~cm}^{-3}$, and $Y_{e}=0.3$, and the reactions (scatterings/absorptions) with neutrons alone are considered.

Figure 1 shows a contour map for the value of $R_{\mathrm{sc} / \mathrm{abs}}$, which is projected against $T_{e}^{\mathrm{eff}}$ and $T_{\mu}^{\mathrm{eff}}$. As discussed above, we believe that the value of $T_{e}^{\mathrm{eff}}$ is very close to the temperature at the neutrinosphere $(3 \mathrm{MeV})$, on the other hand, we take $T_{\mu}^{\text {eff }}$ to the extremely high value, $8 \mathrm{MeV}$, though it may be unlikely to be realized. ${ }^{2}$ This figure clearly shows that a significant part of the drift flux can be attributed to $\nu_{\mu, \tau^{-}} N$ scatterings, for a considerable area in parameter space of $\left(T_{e}^{\mathrm{eff}}, T_{\mu}^{\mathrm{eff}}\right)$. In particular, they are comparable to the effect by the charged-current interactions, when $T_{e}^{\mathrm{eff}}$ is extremely close to the temperature at the neutrinosphere, and this situation may be realized actually. Thus, we cannot neglect the effect of $\nu$-N scatterings even though at the leading order it is canceled between neutrinos and

\footnotetext{
${ }^{2}$ Of course, taking too high value for the effective temperature violates the diffusion approximation, but we believe that it still gives some useful implications.
} 


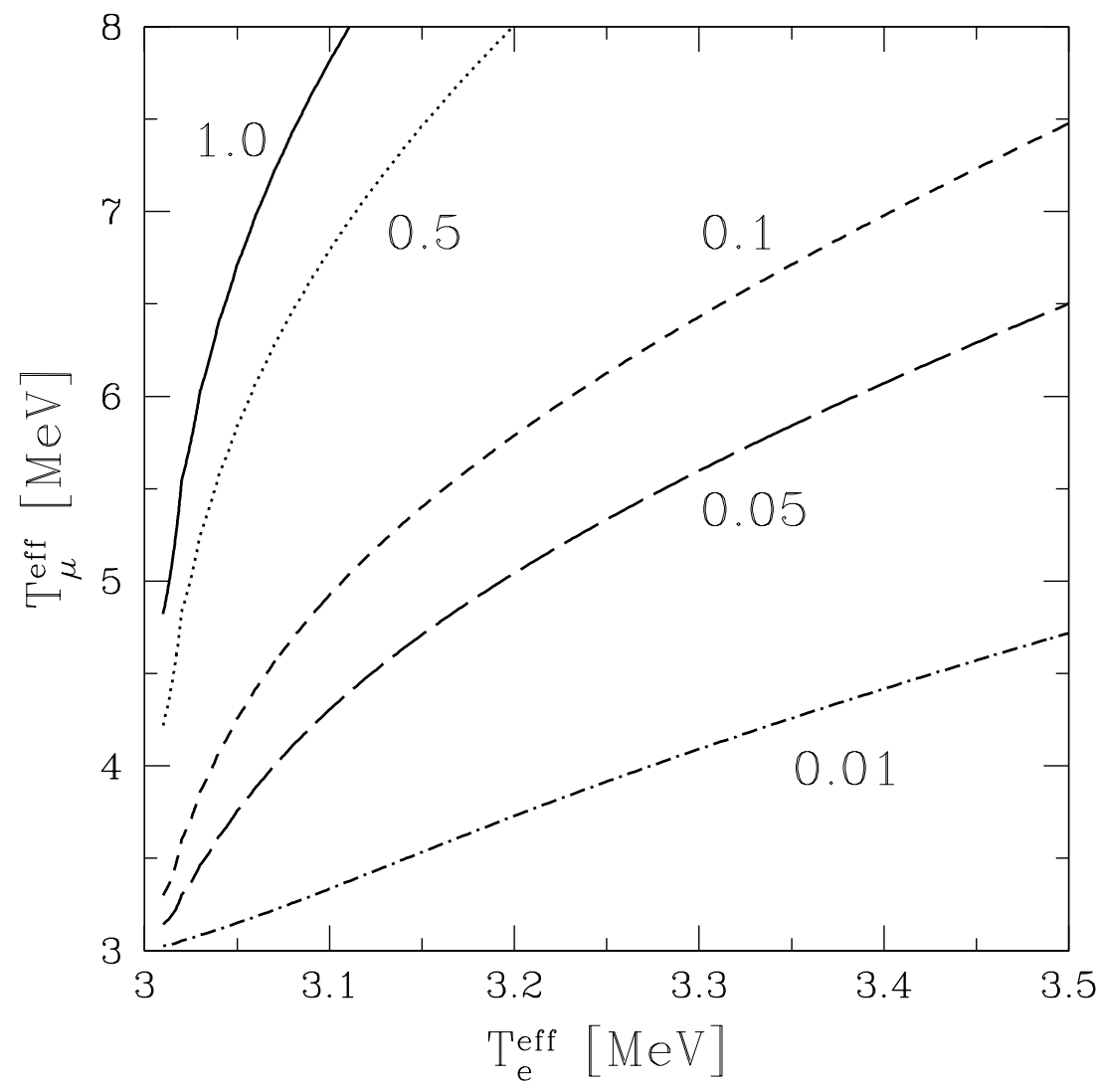

FIG. 1: Contour map for the value of $R_{\mathrm{sc} / \text { abs }}$ projected against $T_{e}^{\mathrm{eff}}$ and $T_{\mu}^{\mathrm{eff}}$.

antineutrinos; it may give considerable contribution to the asymmetric neutrino emission, in addition to the charged-current interactions. Whether we can actually obtain the observed pulsar kicks from this mechanism is beyond the scope of this study, and it is a subject for the future numerical study.

In this paper, we have derived the cross sections of $\nu$ - $N$ scatterings in strong magnetic fields, including weak-magnetism and recoil corrections. Since neutrino interactions are described by theory of the weak interaction which violate the parity, the asymmetric neutrino emission (drift flux) is induced, and it may give the origin of pulsar kicks. The drift flux due to $\nu$-N scatterings is suppressed at $\mathcal{O}\left(\mu_{B} B / m\right)$ level, because the leading contribution is canceled between neutrinos and antineutrinos. However, since the drift flux is proportional to the deviation of neutrino energy density from the value of thermal equilibrium $\delta U_{\nu}$ and it can be large for non-electron neutrinos, it can cancel the small suppression factor of $\mathcal{O}\left(\mu_{B} B / m\right)$. 
We have shown that the drift flux due to $\nu$ - $N$ scatterings may be comparable to the leading term due to beta processes with nucleons. It is expected to give the non-negligible effect for inducing relevant pulsar kicks.

\section{APPENDIX A: DERIVATION OF DIFFERENTIAL CROSS SECTION}

In this section, we derive differential cross section, Eqs. (18)-(23), from more general form, Eqs. (10), (11), and (16), in the case of nondegenerate nucleons.

We do not give the discussion for deriving the detailed form for nucleon response function $S_{s s^{\prime}}$, because it has been already discussed in Ref. [14], and we basically follow their method in deriving the remaining part. Our interest here is concerned with the momentum integral which includes $\boldsymbol{P}$, i.e.,

$$
\int \frac{d^{3} p}{(2 \pi)^{3}} \frac{d^{3} p^{\prime}}{(2 \pi)^{3}}(2 \pi)^{4} \delta^{4}\left(p+k-p^{\prime}-k^{\prime}\right) f_{N}\left(1-f_{N}^{\prime}\right) \boldsymbol{P},
$$

which appears in the second term in the right hand side of Eq. (16). For the term including $\boldsymbol{p}$, we first use $d^{3} p^{\prime}$ to integrate over $\delta^{3}\left(\boldsymbol{p}+\boldsymbol{q}-\boldsymbol{p}^{\prime}\right)$ and then integrate over the azimuthal angle for $\boldsymbol{p}$, resulting in

$$
\frac{\boldsymbol{q}}{2 \pi q} \int_{0}^{\infty} d p p^{3} \int_{-1}^{1} d \mu \mu \delta\left(q_{0}+E-E^{\prime}\right) f_{N}(E)\left[1-f_{N}\left(E^{\prime}\right)\right]
$$

with $\mu=\boldsymbol{p} \cdot \boldsymbol{q} / p q$ and $E^{\prime}=-\mu_{B} B s^{\prime}+(\boldsymbol{p}+\boldsymbol{q})^{2} / 2 m=-\mu_{B} B s^{\prime}+\left(p^{2}+q^{2}+2 p q \mu\right) / 2 m$. Care must be taken to correctly integrate over the energy-conservation delta function with coordinate $\mu$; the integral is only nonzero if the argument of the delta function is zero for $\mu \in(-1,1)$. This condition, $\mu^{2} \leq 1$ is rewritten in the following expression for momentum $p$

$$
p^{2} \geq p_{\min }^{2}=\left[\frac{q_{0}-q^{2} / 2 m-\mu_{B} B\left(s-s^{\prime}\right)}{q / m}\right]^{2} .
$$

Here we change variables from $p$ to $E=-s \mu_{B} B+p^{2} / 2 m$ in the remaining integral; it gives

$$
\begin{gathered}
\frac{m^{3} \boldsymbol{q}}{2 \pi q^{3}} \int_{E_{\min }}^{\infty} d E\left[q_{0}-\frac{q^{2}}{2 m}-\mu_{B} B\left(s-s^{\prime}\right)\right] f_{N}(E)\left[1-f_{N}\left(E+q_{0}\right)\right] \\
=\frac{m \boldsymbol{q}}{q^{2}}\left[q_{0}-\frac{q^{2}}{2 m}-\mu_{B} B\left(s-s^{\prime}\right)\right] S_{s s^{\prime}}\left(q_{0}, q\right) \\
E_{\min }=-\mu_{B} B s+\frac{\left[q_{0}+q^{2} / 2 m-\mu_{B} B\left(s-s^{\prime}\right)\right]^{2}}{4\left(q^{2} / 2 m\right)}
\end{gathered}
$$


Note that the last form of Eq. (A4) contains the integral which is also obtained from the integration of nucleon response function $S_{s s^{\prime}}\left(q_{0}, q\right)$. With the similar manner, the term of Eq. (A1) which includes $\boldsymbol{p}^{\prime}$ is derived in the following form:

$$
\begin{aligned}
& \frac{m^{3} \boldsymbol{q}}{2 \pi q^{3}} \int_{E_{\min }^{\prime}}^{\infty} d E^{\prime}\left[q_{0}+\frac{q^{2}}{2 m}-\mu_{B} B\left(s-s^{\prime}\right)\right] f_{N}\left(E^{\prime}-q_{0}\right)\left[1-f_{N}\left(E^{\prime}\right)\right] \\
& =\frac{m \boldsymbol{q}}{q^{2}}\left[q_{0}+\frac{q^{2}}{2 m}-\mu_{B} B\left(s-s^{\prime}\right)\right] \tilde{S}_{s s^{\prime}}\left(q_{0}, q\right)
\end{aligned}
$$

where

$$
\begin{aligned}
\tilde{S}_{s s^{\prime}}\left(q_{0}, q\right) & =\frac{m^{2}}{2 \pi q} \int_{E_{\min }^{\prime}}^{\infty} d E^{\prime} f_{N}\left(E^{\prime}-q_{0}\right)\left[1-f_{N}\left(E^{\prime}\right)\right] \\
E_{\min }^{\prime} & =-\mu_{B} B s^{\prime}+\frac{\left[q_{0}+q^{2} / 2 m-\mu_{B} B\left(s-s^{\prime}\right)\right]^{2}}{4 q^{2} / 2 m} .
\end{aligned}
$$

From this point on, we show $\tilde{S}_{s s^{\prime}}=S_{s s^{\prime}}$ for nondegenerate nucleons. We first define the dimensionless variables $x^{\prime}=\left(E^{\prime}-\mu_{N}\right) / T$ and $z=q_{0} / T$; the integral yields

$$
\begin{aligned}
\tilde{S}_{s s^{\prime}}\left(q_{0}, q\right) & =\frac{m^{2} T}{2 \pi q} \int_{x_{\min }^{\prime}}^{\infty} d x^{\prime}\left(\frac{1}{e^{x^{\prime}-z}+1}\right)\left(\frac{1}{1+e^{-x^{\prime}}}\right) \\
& =\frac{m^{2} T}{2 \pi q} \frac{1}{1-e^{-z}} \ln \left(\frac{1+e^{-x_{\min }^{\prime}+z}}{1+e^{-x_{\min }^{\prime}}}\right) .
\end{aligned}
$$

Expanding $x_{\min }^{\prime}$ to linear order in $B$ we find

$$
\begin{aligned}
x_{\min }^{\prime} & \simeq x_{0}^{\prime}+\delta x^{\prime}, \\
x_{0}^{\prime} & =\frac{\left(q_{0}+q^{2} / 2 m\right)^{2}}{4 T\left(q^{2} / 2 m\right)}-\frac{\mu_{N}}{T}, \\
\delta x^{\prime} & =\frac{-\mu_{B} B}{2 T}\left[\left(1+\frac{2 m q_{0}}{q^{2}}\right) s+\left(1-\frac{2 m q_{0}}{q^{2}} s^{\prime}\right)\right] .
\end{aligned}
$$

Note that $\delta x^{\prime}$ is identical to $\delta x[$ Eq. (15)] which already appeared in deriving nucleon response function. For $\delta x \ll 1, \tilde{S}_{s s^{\prime}}$ can be written as a sum of $\tilde{S}_{0}$, the zero field value, and $\delta \tilde{S}_{s s^{\prime}}$, the correction due to the magnetic field, i.e.,

$$
\begin{aligned}
\tilde{S}_{s s^{\prime}}\left(q_{0}, q\right) & =\tilde{S}_{0}\left(q_{0}, q\right)+\delta \tilde{S}_{s s^{\prime}}\left(q_{0}, q\right), \\
\tilde{S}_{0}\left(q_{0}, q\right) & =\frac{m^{2} T}{2 \pi q} \frac{1}{1-e^{-z}} \ln \left(\frac{1+e^{-x_{0}^{\prime}+z}}{1+e^{-x_{0}^{\prime}}}\right), \\
\delta \tilde{S}_{s s^{\prime}}\left(q_{0}, q\right) & =-\frac{m^{2} T}{2 \pi q} \frac{\delta x}{\left(1+e^{x_{0}^{\prime}-z}\right)\left(1+e^{-x_{0}^{\prime}}\right)} .
\end{aligned}
$$


In the limit of nondegenerate nucleons with $\mu_{N} / T \ll-1$ and $\exp \left(\mu_{N} / T\right)=\left(2 \pi^{3} / m^{3} T^{3}\right)^{1 / 2} n$, we find

$$
\begin{aligned}
& \frac{1}{1-e^{-z}} \ln \left(\frac{1+e^{-x_{0}^{\prime}+z}}{1+e^{-x_{0}^{\prime}}}\right) \\
& \simeq \frac{1}{\left(1+e^{x_{0}^{\prime}-z}\right)\left(1+e^{-x_{0}^{\prime}}\right)} \\
& \simeq e^{-x_{0}^{\prime}+z}=\exp \left[\frac{\mu_{N}}{T}-\frac{\left(q_{0}-q^{2} / 2 m\right)^{2}}{4 T\left(q^{2} / 2 m\right)}\right] .
\end{aligned}
$$

This last form is the same as that of Eq. (B9) in Ref. [14], which is the $S_{s s^{\prime}}$ limit for

nondegenerate nucleons, guaranteeing the relation $\tilde{S}_{s s^{\prime}}=S_{s s^{\prime}}$ for nondegenerate nucleon limit.

Hence, we obtain the expression for the momentum integral which includes $\boldsymbol{P}$ as follows:

$$
\begin{aligned}
& \int \frac{d^{3} p}{(2 \pi)^{3}} \frac{d^{3} p^{\prime}}{(2 \pi)^{3}}(2 \pi)^{4} \delta^{4}\left(p+k-p^{\prime}-k^{\prime}\right) f_{N}\left(1-f_{N}^{\prime}\right) \boldsymbol{P} \\
& =\frac{2 m \boldsymbol{q}}{q^{2}}\left[q_{0}-\mu_{B} B\left(s-s^{\prime}\right)\right] S_{s s^{\prime}}\left(q_{0}, q\right) .
\end{aligned}
$$

The nuclear response function $S_{s s^{\prime}}\left(q_{0}, q\right)$ can be easily expanded by $\epsilon$ using the same method given in Ref. [14]; leading to Eq. (18). Thus, all the expressions of Eqs. (19)-(23) are also obtained.

\section{ACKNOWLEDGMENTS}

The author is supported by the Research Fellowships of the Japan Society for the Promotion of Science for Young Scientists.

[1] S. Ando and K. Sato, Phys. Rev. D 67, 023004 (2003); hep-ph/0305052.

[2] D. R. Lorimer, M. Bailes, and P. A. Hansen, Mon. Not. R. Astron. Soc. 289, 592 (1997).

[3] B. M. S. Hansen and E. S. Phinney, Mon. Not. R. Astron. Soc. 291, 569 (1997).

[4] Z. Arzoumanian, D. F. Chernoff, and J. M. Cordes, Astrophys. J. 568, 289 (2002).

[5] A. G. Lyne and D. R. Lorimer, Nature 369, 127 (1994).

[6] J. M. Cordes and D. F. Chernoff, Astrophys. J. 505, 315 (1998).

[7] A. Burrows and B. A. Fryxell, Science 258, 430 (1992). 
[8] A. Burrows, J. Hayes, and B. A. Fryxell, Astrophys. J. 450, 830 (1995).

[9] H. T. Janka and E. Müller, Astron. Astrophys. 290, 496 (1994).

[10] H. T. Janka and E. Müller, Astron. Astrophys. 306, 167 (1996).

[11] M. Herant, W. Benz, W. R. Hix, C. L. Fryer, and S. A. Colgate, Astrophys. J. 435, 339 (1994).

[12] A. Burrows and J. Hayes, Phys. Rev. Lett. 76, 352 (1996).

[13] P. Arras and D. Lai, Astrophys. J. 519, 745 (1999).

[14] P. Arras and D. Lai, Phys. Rev. D 60, 043001 (1999).

[15] C. J. Horowitz and G. Li, Phys. Rev. Lett. 80, 3694 (1998).

[16] C. J. Horowitz, Phys. Rev. D 65, 043001 (2002).

[17] Y. Fukuda et al. (The Super-Kamiokande Collaboration), Phys. Rev. Lett. 82, 2644 (1999).

[18] S. Fukuda et al. (The Super-Kamiokande Collaboration), Phys. Lett. B 539, 179 (2002).

[19] K. Eguchi et al. (KamLAND Collaboration), Phys. Rev. Lett. 90, 021802 (2003).

[20] A. S. Dighe and A. Y. Smirnov, Phys. Rev. D 62, 033007 (2000).

[21] A. Kusenko and G. Segrè, Phys. Rev. Lett. 77, 4872 (1996). 